\title{
Facile Oxidations under Flow Conditions in Fixed-Bed Reactors
}

Polymer-Supported

Synthesis

Key words

inductive heating

oxidation

flow conditions

metal oxides

flow reactor filled with magnetic nanoparticles and solid oxidant

$$
\text { inductor }
$$
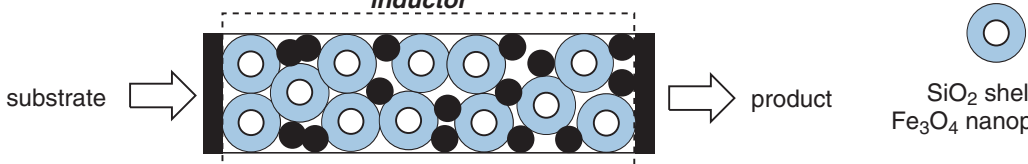

$\mathrm{SiO}_{2}$ shelled $\mathrm{Fe}_{3} \mathrm{O}_{4}$ nanoparticle

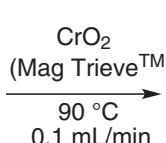

$0.1 \mathrm{~mL} / \mathrm{min}$

1<smiles>O=C1c2ccccc2C(=O)c2ccccc21</smiles><smiles>CCC#Cc1ccccc1</smiles>

3

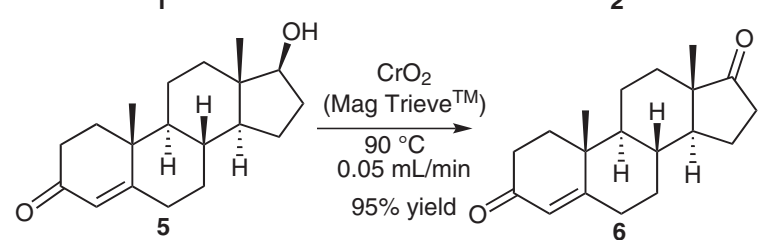<smiles>COc1ccc2cc(C(C)O)ccc2c1</smiles><smiles>OC/C=C/c1ccccc1</smiles>

13<smiles>COc1ccc(CN)cc1OC</smiles>

17
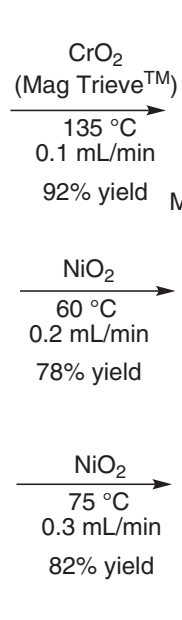<smiles>CCC(O)c1ccccc1</smiles><smiles>c1ccc2c(c1)CCOC2</smiles>

11

10<smiles>O=C/C=C/c1ccccc1</smiles>

14<smiles>COc1ccc(C#N)cc1OC</smiles>

18<smiles>COc1ccccc1CN</smiles>

15

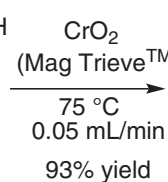<smiles>O=CC#Cc1ccccc1</smiles><smiles>CCC(=O)c1ccccc1</smiles>

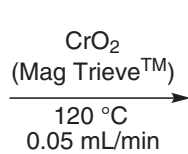<smiles>O=C1OCCc2ccccc21</smiles>

12

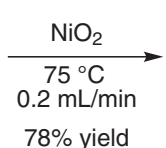<smiles>COc1ccccc1C#N</smiles>

16

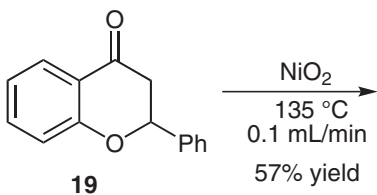<smiles>O=c1cc(-c2ccccc2)oc2ccccc12</smiles>

20

Significance: Flow oxidation with inductive heating inside flow reactors was described. The oxidation reactions of various substrates were carried out in glass flow reactors charged with superparamagnetic nanoparticles and solid oxidants, which were exposed to a magnetic field with an inductive frequency of $25 \mathrm{kHz}$ at 40 psi backpressure to afford the corresponding products.
Comment: The flow oxidation of substrates $\mathbf{9 , 1 1}$ or $\mathbf{1 9}$ was performed by using a PEEK (polyether ether ketone) reactor at 100 psi backpressure. The authors previously reported the first application of ferromagnetic materials like magnetic nanoparticles $\mathrm{Fe}_{3} \mathrm{O}_{4}$ coated with a silica shell (10-40 nm) as heating media in flow reactions (Angew. Chem. Int. Ed. 2008, 47, 8950).

SYNFACTS Contributors: Yasuhiro Uozumi, Yoichi M. A. Yamada, Maki Minakawa 